\title{
THE USAGE OF TREFFTZ FUNCTIONS AND PICARD'S ITERATION FOR SOLVING DIFFERENT PROBLEMS OF A TWO-DIMENSIONAL WAVE EQUATION
}

\author{
Patrycja Krzyszkowska ${ }^{1}$, Artur Maciag ${ }^{2}$, Magdalena Walaszczyk ${ }^{3}$ \\ ${ }^{1,3}$ Faculty of Mechatronics and Machine Design, Kielce University of Technology \\ Kielce, Poland \\ ${ }^{2}$ Faculty of Management and Computer Modelling, Kielce University of Technology \\ Kielce, Poland \\ Ipatrycja.mazurczak@gmail.com, ${ }^{2}$ maciag@tu.kielce.pl, ${ }^{3}$ magda.kisiel2@wp.pl
}

\begin{abstract}
The paper presents a method of solving two-dimensional wave equations which describe vibrations of the membrane with variable thickness and with damping. The differential operator is decomposed into two parts. The first one describes vibrations of the membrane with constant thickness without damping. The second contains the rest of the original operator and is treated as inhomogeneity for the first one. Picard's iterations are used to calculate a successive approximation of the exact solution. Trefftz functions (wave polynomials) are used to solve the problem in each iteration. The presented examples show the usefulness of the method. The approach described in this paper can be used also for solving nonlinear problems for a wave equation.
\end{abstract}

Keywords: wave equation, Trefftz functions, Picard's iteration

\section{Introduction}

The wave equation describes commonly occurring vibrations. One-dimensional (one spatial variable) wave equation describes vibration of strings. Vibrations of membranes are described by a two-dimensional wave equation. Sound waves can be described by a three-dimensional wave equation. This equation can be solved by means of different methods. Firstly, analytical methods should be mentioned, such as integral transformations, a variable separation, the Green function etc. Unfortunately, analytical methods can be applied only in case of a simple shape of the domain. Next, FEM or BEM can be used for solving a wave equation. In spite of the high usefulness of the method for solving different problems, it cannot be used for solving inverse problems. Recently there has been a rapid development of the Trefftz functions method, which can be used for solving different problems described by partial differential equations. This method is especially effective for solving inverse problems. The Trefftz method was first described by Erich Trefftz in 1926 in the paper [1]. Next, a lot of scientists developed this theory, including Herrera, Jirousek, Kupradze, Leon, Sabina, Zieliński and Zienkiewicz [2-6]. 
The paper [7] is the first work concerning Trefftz functions (heat polynomials) where the time was treated as a continuous variable. Heat functions (Trefftz functions for heat conduction equation) have been described or used in the papers [8-11]. Wave polynomials are described and used in the papers [12-15]. Also, several monographs have been written concerning Trefftz functions [16-20].

\section{Presentation of the method}

Let us consider the equation:

$$
A u(x, t)=f(x, t) \text { for }(x, t) \in \Omega \times\langle 0, \infty),
$$

where $A$ is a partial differential operator and $\Omega$ is a bounded subset of $R^{n}$. A direct problem equation (1) should be completed by the initial boundary condition:

$$
\begin{gathered}
I u(\boldsymbol{x}, 0)=h(\boldsymbol{x}) \quad \text { for }(\boldsymbol{x}) \in \Omega, \\
B u(\boldsymbol{x}, t)=g(\boldsymbol{x}, t) \text { for }(\boldsymbol{x}, t) \in \partial \Omega \times\langle 0, \infty) .
\end{gathered}
$$

In particular the operator $A$ can be nonlinear. Let us assume that the operator $A$ can be decomposed into a simple (the solution can be found easily) linear part $L$ and the rest according to formula:

$$
A=L+N
$$

Then equation (1) has the form:

$$
L u(\boldsymbol{x}, t)=f(\boldsymbol{x}, t)-N u(\boldsymbol{x}, t) \text { for }(\boldsymbol{x}, t) \in \Omega \times\langle 0, \infty) .
$$

The method of successive approximation (known as Picard's iteration) can be used to solve equation (5):

$$
\begin{gathered}
L u^{(k)}(\boldsymbol{x}, t)=f(\boldsymbol{x}, t)-N u^{(k-1)}(\boldsymbol{x}, t) \quad \text { for }(\boldsymbol{x}, t) \in \Omega \times\langle 0, \infty), \\
I u^{(k)}(\boldsymbol{x}, 0)=h(x) \quad \text { for }(\boldsymbol{x}) \in \Omega, \\
B u^{(k)}(\boldsymbol{x}, t)=g(\boldsymbol{x}, t) \quad \text { for }(\boldsymbol{x}, t) \in \partial \Omega \times\langle 0, \infty) .
\end{gathered}
$$

For $k=1$ we take:

$$
L u^{(1)}(\boldsymbol{x}, t)=f(\boldsymbol{x}, t) .
$$

If the Trefftz functions for linear operator $L$ are known, the solution $u(\boldsymbol{x})$ can be approximated in each iteration by (omitting index $k$ ):

$$
u(\boldsymbol{x}, t) \approx w(\boldsymbol{x}, t)=\sum_{n=1}^{N} c_{n} V_{n}(\boldsymbol{x}, t)+w_{p}(\boldsymbol{x}, t),
$$


where $c_{n}$ are coefficients, $V_{n}$ are Trefftz functions for linear operator $L$ and $w_{p}$ is the particular solution of nonhomogeneous equation (6). Coefficients $c_{n}$ are determined by initial and boundary conditions. Suitable functional is built, which describes error of fulfilling the initial and boundary conditions by approximate solutions (in least square sense). The inhomogeneity can be expanded into the Taylor series and approximated by sum of monomials. Therefore, the inverse operator $L^{-1}$ for monomials has to be known.

\section{Examples}

In this section examples of the presented method will be considered. To check the quality of the method the approximation will be compared with the exact solution.

\subsection{Membrane with variable thickness}

\subsubsection{Example of the problem with known exact solution}

Let us consider a forced vibration of membrane with variable thickness described by equation:

$$
\frac{\partial^{2} u}{\partial t^{2}}=\frac{\partial}{\partial x}\left(f(x, y) \frac{\partial u}{\partial x}\right)+\frac{\partial}{\partial y}\left(f(x, y) \frac{\partial u}{\partial y}\right)+Q(x, y, t)
$$

where:

$$
\begin{gathered}
Q(x, y, t)=\frac{1}{2} x^{3}-x^{3} t^{2}-4 x^{2} y^{2}+5 x^{2} y-2 x^{2} y t^{2}-\frac{11}{4} x^{2}+\frac{11}{2} x^{2} t^{2} \\
+5 x y^{2}+-2 x y^{2} t^{2}-6 x y+4 x y t^{2}+\frac{9}{4} x-\frac{9}{2} x t^{2}+\frac{1}{2} y^{3} \\
-y^{3} t^{2}-\frac{11}{4} y^{2}+\frac{11}{2} y^{2} t^{2}+\frac{9}{4} y+-\frac{9}{2} y t^{2}
\end{gathered}
$$

and

$$
f(x, y)=1-\frac{1}{4} x-\frac{1}{4} y
$$

with conditions:

$$
\begin{gathered}
u(x, y, 0)=u_{0}(x, y)=x(x-1) y(y-1), \\
\frac{\partial u}{\partial t}(x, y, 0)=0, \\
u(0, y, t)=u(1, y, t)=u(x, 0, t)=u(x, 1, t)=0 .
\end{gathered}
$$


The exact solution of the problem described by (11)-(16) has the form:

$$
u(x, y, t)=x(x-1) y(y-1)\left(1-2 t^{2}\right) .
$$

Equation (11) can be converted to the form according to (4):

$\frac{\partial^{2} u}{\partial t^{2}}-\frac{\partial^{2} u}{\partial x^{2}}-\frac{\partial^{2} u}{\partial y^{2}}=-\frac{1}{4} \frac{\partial u}{\partial x}-\left(\frac{x+y}{4}\right) \frac{\partial^{2} u}{\partial x^{2}}-\frac{1}{4} \frac{\partial u}{\partial y}-\left(\frac{x+y}{4}\right) \frac{\partial^{2} u}{\partial y^{2}}+Q(x, y, t)$.

In equation (18) the operators $L u$ and $N u$ have a form respectively:

$$
\begin{gathered}
L u=\frac{\partial^{2} u}{\partial t^{2}}-\frac{\partial^{2} u}{\partial x^{2}}-\frac{\partial^{2} u}{\partial y^{2}} \\
N u=-\frac{1}{4} \frac{\partial u}{\partial x}-\left(\frac{x+y}{4}\right) \frac{\partial^{2} u}{\partial x^{2}}-\frac{1}{4} \frac{\partial u}{\partial y}-\left(\frac{x+y}{4}\right) \frac{\partial^{2} u}{\partial y^{2}}+Q(x, y, t) .
\end{gathered}
$$

The exact solution is approximated by:

$$
u(x, y, t) \approx w(x, y, t)=\sum_{n=1}^{N} c_{n} V_{n}(x, y, t)+w_{p}(x, y, t) .
$$

The Trefftz functions $V_{n}$ are wave polynomials described in [12]. In the first step of iteration we have:

$$
\frac{\partial^{2} u^{(1)}}{\partial t^{2}}-\frac{\partial^{2} u^{(1)}}{\partial x^{2}}-\frac{\partial^{2} u^{(1)}}{\partial y^{2}}=Q(x, y, t) .
$$

Then:

$$
u^{(1)}(x, y, t) \approx w^{(1)}(x, y, t)=\sum_{n=1}^{N} c_{n}^{(1)} V_{n}^{(1)}(x, y, t)+w_{p}^{(1)}(x, y, t) .
$$

The particular solution is calculated according to formula:

$$
w_{p}^{(1)}(x, y, t)=L^{-1}(Q(x, y, t)) .
$$

In the considered case the source $Q(x, y, t)$ has a form of a polynomial. Therefore, the inverse operator for monomials has to be known. Let us denote $r_{k m l}=L^{-1}\left(x^{k} y^{m}\right.$ $\left.t^{l}\right)$, where $k, l, m=0,1, \ldots$. Suitable formulas are presented in the paper [15]:

$$
\begin{aligned}
& r_{k m l}^{1}=-\frac{x^{k+2} y^{m} t^{l}-l(l-1) r_{(k+2) m(l-2)}^{1}+m(m-1) r_{(k+2)(m-2) l}^{1}}{(k+2)(k+1)}, \\
& r_{k m l}^{2}=-\frac{x^{k} y^{m+2} t^{l}-l(l-1) r_{k(m+2)(l-2)}^{2}+k(k-1) r_{(k-2)(m+2) l}^{2}}{(m+2)(m+1)}, \\
& r_{k m l}^{3}=\frac{x^{k} y^{m} t^{l+2}+k(k-1) r_{(k-2) m(l+2)}^{3}+m(m-1) r_{k(m-2)(l+2)}^{3}}{(l+2)(l+1)} .
\end{aligned}
$$


For calculations we take:

$$
L^{-1}\left(x^{k} y^{m} t^{l}\right)=\frac{1}{3}\left(r^{1}\left(x^{k} y^{m} t^{l}\right)+r^{2}\left(x^{k} y^{m} t^{l}\right)+r^{3}\left(x^{k} y^{m} t^{l}\right)\right) .
$$

Coefficients $c_{n}$ are chosen so that the mean square error of fulfilling initial and boundary conditions by an approximate solution is minimized. In the next step the equation has the form:

$$
\begin{aligned}
\frac{\partial^{2} u^{(2)}}{\partial t^{2}}-\frac{\partial^{2} u^{(2)}}{\partial x^{2}} & -\frac{\partial^{2} u^{(2)}}{\partial y^{2}}= \\
& =-\frac{1}{4} \frac{\partial u^{(1)}}{\partial x}-\left(\frac{x+y}{4}\right) \frac{\partial^{2} u^{(1)}}{\partial x^{2}}-\frac{1}{4} \frac{\partial u^{(1)}}{\partial y}-\left(\frac{x+y}{4}\right) \frac{\partial^{2} u^{(1)}}{\partial y^{2}}+ \\
& +Q(x, y, t) .
\end{aligned}
$$

Now an approximate solution is given in the form:

$$
u^{(2)}(x, y, t) \approx w^{(2)}(x, y, t)=\sum_{n=1}^{N} c_{n}^{(2)} V_{n}^{(2)}(x, y, t)+w_{p}^{(2)}(x, y, t) .
$$

$c_{n}$ and the particular solution are calculated as before.

Because the exact solution is known, the quality of the approximation can be checked using two kinds of the error. The first one is calculated for point $x=y=0.5$ (middle point of the membrane):

$$
E_{m}=\sqrt{\frac{\int_{0}^{\Delta t}[u(0.5,0.5, t)-w(0.5,0.5, t)]^{2} d t}{\int_{0}^{\Delta t}[u(0.5,0.5, t)]^{2} d t}} \cdot 100 \% .
$$

The second error is calculated for the entire domain:

$$
E=\sqrt{\frac{\int_{0}^{1} \int_{0}^{1} \int_{0}^{1}[u(x, y, t)-w(x, y, t)]^{2} d x d y d t}{\int_{0}^{1} \int_{0}^{1} \int_{0}^{1}[u(x, y, t)]^{2} d x d y d t}} \cdot 100 \%
$$

The values of the errors $E_{m}$ and $E$, depending on the number of iterations and the number of wave polynomials $n$, used in approximation are presented in Table 1 and Table 2 respectively.

In general the error of approximation decreases after each iteration. After the first step the error is relatively big because an incomplete equation is taken into account. We can also observe a significant improvement in approximation when the number of wave polynomials is greater than 36 . The value of the error after five iterations (both for point $x=y=0.5$ and for entire domain) is smaller than $0.3 \%$, which proves a very good quality of the approximation. 
A mean relative error of approximation $\left(E_{m}[\%]\right)$ for $x=y=0.5$

\begin{tabular}{|c|c|c|c|}
\hline \multirow{2}{*}{ Number of polynomials } & \multicolumn{3}{|c|}{ Number of iterations } \\
\cline { 2 - 4 } & 1 & 3 & 5 \\
\hline 25 & 6.87 & 3.94 & 4.46 \\
\hline 36 & 10.92 & 0.27 & 0.19 \\
\hline 81 & 14.53 & 0.27 & 0.11 \\
\hline
\end{tabular}

Table 2

A mean relative error of approximation $(E[\%])$ in the entire domain

\begin{tabular}{|c|c|c|c|}
\hline \multirow{2}{*}{ Number of polynomials } & \multicolumn{3}{|c|}{ Number of iterations } \\
\cline { 2 - 4 } & 1 & 3 & 5 \\
\hline 25 & 15.31 & 12.10 & 12.69 \\
\hline 36 & 11.45 & 1.06 & 0.45 \\
\hline 81 & 14.15 & 1.15 & 0.28 \\
\hline
\end{tabular}

Figure 1 shows the exact solution for $x=0.5, y=0.5$ (solid line) and the approximation by 81 wave polynomials (dotted line) after: a) 1, b) 2, c) 5 iterations.

a)

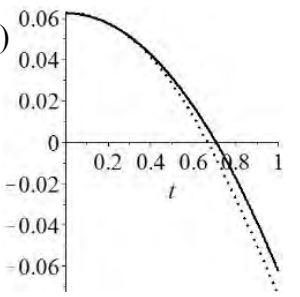

b)

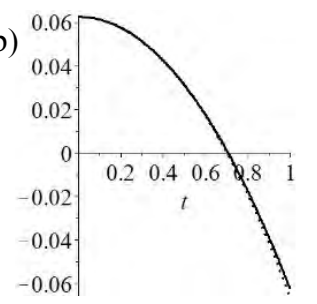

c)

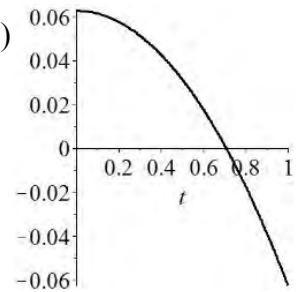

Fig. 1. The exact solution (solid line) for $\mathrm{x}=0.5, \mathrm{y}=0.5$ and the approximation by 81 wave polynomials (dotted line) after: a) 1, b) 2, c) 5 iterations

Figure 2 shows the exact solution, approximation by 81 wave polynomials after 5 iterations and the error of approximation for $t=0$.
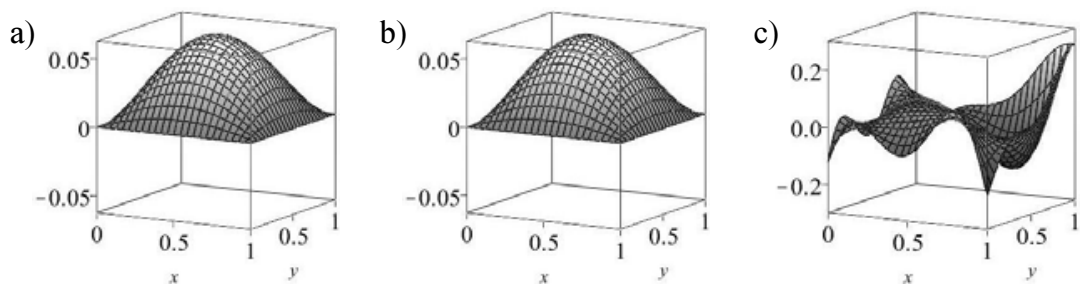

Fig. 2. The solution for $t=0$ : a) exact, b) approximation after 5 iterations,

c) error of approximation

Figures 1 and 2 show a very good accuracy of the approximation. 


\subsubsection{Example of the problem without an exact solution}

As a second example we consider free vibrations of the membrane with variable thickness. The problem is described by equation:

$$
\frac{\partial^{2} u}{\partial t^{2}}=\frac{\partial}{\partial x}\left(f(x, y) \frac{\partial u}{\partial x}\right)+\frac{\partial}{\partial y}\left(f(x, y) \frac{\partial u}{\partial y}\right)
$$

where:

$$
f(x, y)=1-\frac{1}{4} x-\frac{1}{4} y
$$

and conditions:

$$
\begin{gathered}
u(x, y, 0)=u_{0}(x, y)=x(x-1) y(y-1), \\
\frac{\partial u}{\partial t}(x, y, 0)=0, \\
u(0, y, t)=u(1, y, t)=u(x, 0, t)=u(x, 1, t)=0 .
\end{gathered}
$$

Equation (33) can be converted to the form:

$$
\frac{\partial^{2} u}{\partial t^{2}}-\frac{\partial^{2} u}{\partial x^{2}}-\frac{\partial^{2} u}{\partial y^{2}}=-\frac{1}{4} \frac{\partial u}{\partial x}-\left(\frac{x+y}{4}\right) \frac{\partial^{2} u}{\partial x^{2}}-\frac{1}{4} \frac{\partial u}{\partial y}-\left(\frac{x+y}{4}\right) \frac{\partial^{2} u}{\partial y^{2}}
$$

In equation (38) we have:

$$
\begin{gathered}
L u=\frac{\partial^{2} u}{\partial t^{2}}-\frac{\partial^{2} u}{\partial x^{2}}-\frac{\partial^{2} u}{\partial y^{2}} \\
N u=-\frac{1}{4} \frac{\partial u}{\partial x}-\left(\frac{x+y}{4}\right) \frac{\partial^{2} u}{\partial x^{2}}-\frac{1}{4} \frac{\partial u}{\partial y}-\left(\frac{x+y}{4}\right) \frac{\partial^{2} u}{\partial y^{2}} .
\end{gathered}
$$

The method of the solution is similar to the previous example. The approximation has the form:

$$
u(x, y, t) \approx w(x, y, t)=\sum_{n=1}^{N} c_{n} V_{n}(x, y, t)+w_{p}(x, y, t),
$$

where:

$$
\begin{aligned}
w_{p}^{(k+1)}(x, y, t) & =-L^{-1}\left(\frac{1}{4} \frac{\partial w^{(k)}}{\partial x}+\left(\frac{x+y}{4}\right) \frac{\partial^{2} w^{(k)}}{\partial x^{2}}+\frac{1}{4} \frac{\partial w^{(k)}}{\partial y}\right. \\
& \left.+\left(\frac{x+y}{4}\right) \frac{\partial^{2} w^{(k)}}{\partial y^{2}}\right) .
\end{aligned}
$$


In the considered case the thickness of the membrane depends on variables $x$ and $y$. Therefore, the solution of the problem is asymmetric with respect to both lines: $x=0.5$ and $y=0.5$. This effect is shown in Figure 3. Here the approximation by 81 wave polynomials after 1 and 5 iterations for $t=0.5$ were used. In the upper drawings the membrane is seen from axis $x$, while in the lower drawings it is seen from axis $y$.

a)
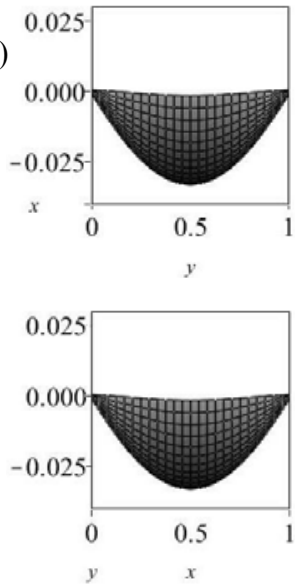

b)
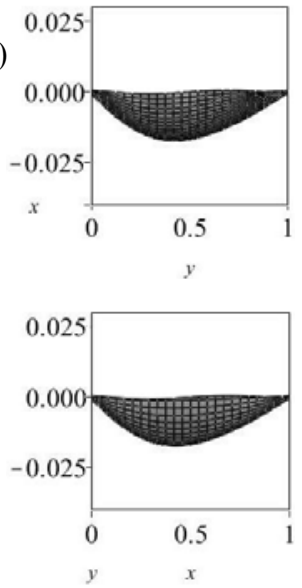

Fig. 3. The solution for $t=0.5$ after: a) 1 and b) 5 iterations. The upper drawings show the membrane from the axis $x$, while the lower from the axis $y$

Figure 3 shows that that the asymmetry is noticeable after 5 iterations.

\subsection{Membrane with damping}

\subsubsection{Vibration with damping coefficient $H=2$}

As the next example let us consider a wave equation which describes vibrations of the membrane with damping coefficient $H=2$ :

$$
\frac{\partial^{2} u}{\partial t^{2}}+H \frac{\partial u}{\partial t}=\frac{\partial^{2} u}{\partial x^{2}}+\frac{\partial^{2} u}{\partial y^{2}}
$$

initial conditions:

$$
\begin{gathered}
u(x, y, 0)=100 \sin \left(\frac{1}{2} x+\frac{\sqrt{3}}{2} y\right), \\
\frac{\partial u}{\partial t}(x, y, 0)=-100 \sin \left(\frac{1}{2} x+\frac{\sqrt{3}}{2} y\right),
\end{gathered}
$$


and boundary conditions:

$$
\begin{gathered}
u(0, y, t)=100 e^{-t} \sin \left(\frac{\sqrt{3}}{2} y\right) \text { for } t>0, \\
u(x, 0, t)=100 e^{-t} \sin \left(\frac{1}{2} x\right) \text { for } t>0, \\
u(x, 1, t)=100 e^{-t} \sin \left(\frac{1}{2} x+\frac{\sqrt{3}}{2}\right) \text { for } t>0, \\
u(1, y, t)=100 e^{-t} \sin \left(\frac{1}{2}+\frac{\sqrt{3}}{2} y\right) \text { for } t>0 .
\end{gathered}
$$

The exact solution for the problem described by (43) and conditions (44)-(49) has the form:

$$
u(x, y, t)=100 e^{-t} \sin \left(\frac{1}{2} x+\frac{\sqrt{3}}{2} y\right)
$$

Similarly as before, equation (43) can be converted to the form:

$$
\frac{\partial^{2} u}{\partial t^{2}}-\frac{\partial^{2} u}{\partial x^{2}}-\frac{\partial^{2} u}{\partial y^{2}}=-H \frac{\partial u}{\partial t}
$$

In equation (51) the operators $L$ and $N$ are given by formulas:

$$
\begin{gathered}
L u=\frac{\partial^{2} u}{\partial t^{2}}-\frac{\partial^{2} u}{\partial x^{2}}-\frac{\partial^{2} u}{\partial y^{2}} \\
N u=-H \frac{\partial u}{\partial t} .
\end{gathered}
$$

The solution of the considered problem follows practically the same way as before. The exact solution is approximated by:

$$
u(x, y, t) \approx w(x, y, t)=\sum_{n=1}^{N} c_{n} V_{n}(x, y, t)+w_{p}(x, y, t) .
$$

The particular solution is calculated as:

$$
w_{p}^{(k+1)}(x, y, t)=-L^{-1}\left(H \frac{\partial w^{(k)}}{\partial t}\right)
$$


Because the exact solution is known, the quality of the approximation can be checked by two kinds of error, as in the first example. The values of the error $E_{m}$ depending on the number of iterations and the number of wave polynomials $n$ used in approximation for the middle of the membrane and in the entire domain are presented in Table 3 and Table 4, respectively. In the first iteration the errors are the biggest because it is a solution of a homogeneous equation. The increase in the number of iterations results in reducing the errors. Also using more polynomials causes a decrease in the error of approximation. The best results were observed for 81 polynomials after 5 iterations for both types of errors. Additionally, a mean relative error of approximation in the entire domain is smaller than in the middle of the membrane.

Table 3

A mean relative error of approximation $\left(E_{m}[\%]\right)$ for $x=y=0.5$

\begin{tabular}{|c|c|c|c|}
\hline \multirow{2}{*}{ Number of polynomials } & \multicolumn{3}{|c|}{ Number of iterations } \\
\cline { 2 - 4 } & 1 & 3 & 5 \\
\hline 16 & 1.81 & 0.39 & 0.45 \\
\hline 49 & 5.82 & 0.78 & 0.22 \\
\hline 81 & 7.86 & 0.86 & 0.19 \\
\hline
\end{tabular}

Table 4

A mean relative error of approximation $(E[\%])$ in the entire domain

\begin{tabular}{|c|c|c|c|}
\hline \multirow{2}{*}{ Number of polynomials } & \multicolumn{3}{|c|}{ Number of iterations } \\
\cline { 2 - 4 } & 1 & 3 & 5 \\
\hline 16 & 0.94 & 0.71 & 0.42 \\
\hline 49 & 2.93 & 0.39 & 0.13 \\
\hline 81 & 3.84 & 0.46 & 0.10 \\
\hline
\end{tabular}

Figure 4 shows the exact solution, approximation by 81 wave polynomials after 5 iterations and the error of approximation for $t=0$. It is clearly seen that the method gives very good results, as the approximation is very close to the accurate solution and the error oscillates around 0 .

a)

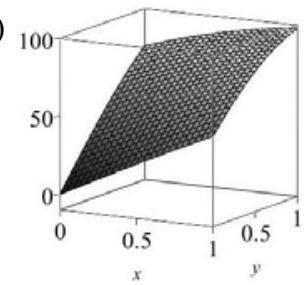

b)

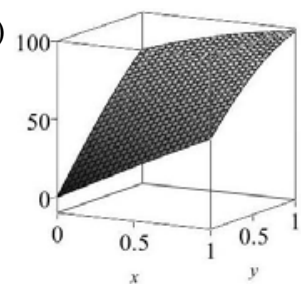

c)

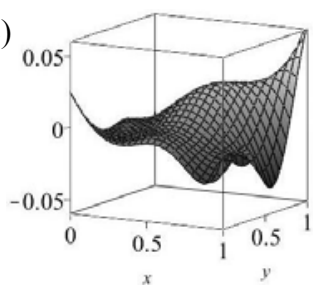

Fig. 4. The solution for $t=0$ : a) exact, b) approximation after 5 iterations, c) relative error of approximation related to the maximum value of the function in entire domain 


\subsubsection{Example of the problem for different $H$}

In this example the damping of the membrane depends on the damping coefficient $H$. A wave equation, which describes vibrations of the membrane with different damping coefficient, is given in the form:

$$
\frac{\partial^{2} u}{\partial t^{2}}+H \frac{\partial u}{\partial t}=\frac{\partial^{2} u}{\partial x^{2}}+\frac{\partial^{2} u}{\partial y^{2}}
$$

The approximation is obtained similarly as in the previous example. Figure 5 shows the effect of damping coefficient on the approximated solution by 100 wave polynomials with different $H$ for $x=0.5, y=0.5$.
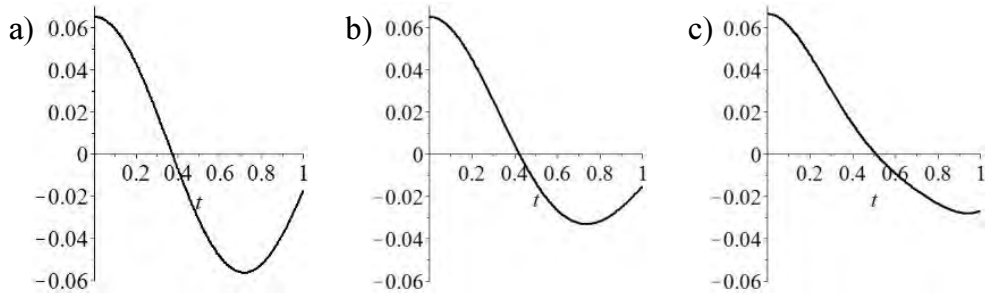

Fig. 5. The solution for $x=0.5, y=0.5$ for: a) $H=0.5$, b) $H=2$, c) $H=4$, after 5 iterations

The damping coefficient affects the rate of damping of the membrane. Figure 5 shows that a higher damping coefficient causes a faster disappearance of the vibration of the membrane.

\section{Conclusions}

The Trefftz functions are extremely useful in solving problems described by partial differential equations. In this paper these functions have been used to solve wave equations which describe the vibrations of the membrane. An advantage of the presented method is its mathematical simplicity. Successive approximations are obtained using Picard's iteration. An approximate solution consists of a linear combination of Trefftz functions and a particular solution for a nonhomogeneous equation. The inhomogeneity is related to the variable thickness of the membrane and damping. The presented method gives very good results for a membrane of varying thickness as well as a membrane with damping. In both cases the error decreases with the increasing number of polynomials and iterations. A satisfactory approximation is obtained already in the third step even for a small number of wave polynomials. In this paper only linear problems have been described. However, the same method can be used for nonlinear cases. 


\section{References}

[1] Trefftz E., Ein Gegenstueck zum Ritz'schen Verfahren, Proceedings of 2nd International Congress of Applied Mechanics, Zurich 1926, 131-137.

[2] Herrera I., Sabina F., Connectivity as an alternative to boundary integral equations: Construction of bases, Appl. Math. Phys. Sc. 1978, 75/5, 2059-2063.

[3] Jirousek J., Basis for development of large finite elements locally satisfying all fields equations, Comp. Meth. Appl. Mech. Eng. 1978, 65-92.

[4] Jirousek J., Zieliński A.P., Rabemantantsoa H., Venkatesh A., Survey of Trefftz-type element formulations, Computers and Structures 1997, 63/2, 225-242.

[5] Kupradze V.D., Three-dimensional Problems of the Mathematical Theory of Elasticity and Thermoelasticity, North-Holland Publ. Cmp., Amsterdam 1989.

[6] Zieliński A.P., Zienkiewicz O.C., Generalized finite element analysis with T-complete boundary solution functions, Int. J. Numer. Meth. Eng. 1985, 21, 509-528.

[7] Rosenbloom P.C., Widder D.V., Expansion in terms of heat polynomials and associated functions, Trans. Am. Math. Soc. 1956, 92, 220-266.

[8] Ciałkowski M.J., Solution of inverse heat conduction problem with use new type of finite element base functions, [in:] Proceedings of the International Symposium on Trends in Continuum Physics, B.T. Maruszewski, W. Muschik, A. Radowicz (eds.), World Scientific Publishing, Singapore, New Jersey, London, Hong Kong 1999, 64-78.

[9] Ciałkowski M.J., Frąckowiak A., Grysa K., Solution of a stationary inverse heat conduction problems by means of Trefftz non-continuous method, Int. J. of Heat Mass Transfer 2007, 50, 2170-2181.

[10] Piasecka M., Maciejewska B., The study of boiling heat transfer in vertically and horizontally oriented rectangular minichannels and the solution to the inverse heat transfer problem with the use of the Beck method and Trefftz functions, Experimental Thermal and Fluid Science 2012, 38, 19-32.

[11] Piasecka M., Maciejewska B., Enhanced heating surface application in a minichannel flow and the use of the FEM and Trefftz functions for the solution of inverse heat transfer problem, Experimental Thermal and Fluid Science 2013, 44, 23-33.

[12] Maciag A., Wauer J., Solution of the two-dimensional wave equation by using wave polynomials, Journal of Engineering Mathematics 2005, 51/4, 339-350.

[13] Maciag A., Solution of the three-dimensional wave polynomials, Mathematical Problems in Engineering 2005, 5, 583-598.

[14] Maciag A., The usage of wave polynomials in solving direct and inverse problems for twodimensional wave equation, Int. J. Numer. Meth. Biomed. Eng. 2011, 27/7, 1107-1125.

[15] Maciag A., Wauer J., Wave polynomials for solving different types of two-dimensional wave equations, Computer Assisted Mechanics and Engineering Sciences (CAMES) 2005, 12, 363-378.

[16] Ciałkowski M.J., Frąckowiak A., Heat Functions and Their Application for Solving Heat Transfer and Mechanical Problems, Poznań University of Technology Publishers, Poznań 2000 (in Polish).

[17] Qing-Hua Qin, The Trefftz Finite and Boundary Element Method, WITPress, Southampton, Boston 2000.

[18] Li Z-C., Qiu Lu T-T., Hu H-Y., Cheng H-D., The Trefftz and Collocation Methods, WIT Press, Southampton, Boston 2008.

[19] Kołodziej J.A., Zieliński A.P., Boundary Collocation Techniques and Their Application in Engineering, WIT Press, Southampton, Boston 2009.

[20] Grysa K., Trefftz Functions and Their Applications in Solving the Inverse Problems, Kielce University of Technology Publishers, Kielce 2010 (in Polish). 